\title{
Research on the Testing Method of Projectile's Interior Ballistics Parameters in Nose to Tail Loading State
}

\author{
Hang $\mathrm{Yu}^{1}$, Kong Deren ${ }^{1}$, Shang Fei ${ }^{1}$ and Liu Deqiu ${ }^{2}$ \\ ${ }^{1}$ School of Mechanical Engineering, Nanjing University of Science \& \\ Technology, Nanjing 210094, China \\ ${ }^{2}$ Centre of Arms Experiment, Baicheng 137001, China \\ hangyu_njust@163.com,derenkongnj@sina.com,11526291@qq.com, \\ LDQDL1@sina.com
}

\begin{abstract}
According to the fact that the $30 \mathrm{~mm}$ diameter projectile in nose to tail loading state is driven by base pressure and nose pressure coming from propellant gas, we propose a storage measurement method to obtain the signal of projectile's base pressure, nose pressure and motion acceleration. By this method, the projectile's interior ballistics characteristic parameters can be achieved. The overall scheme of the storage measurement device is designed. Based on the inertial force correction, the structure design of the simulation test projectile as well as the selection of pressure sensors, the pressure measurement model is established. We test projectile's acceleration and the force act on the force sensors simultaneously, and process the acquired signals with the correction model to obtain the actual base and nose pressure. The test circuit module is designed to process and store the signal form sensors. In the end of paper, several key technologies are discussed in the design progress of the storage measurement device, including mechanical design of the pressure measurement part, the test circuit module miniaturization methods, the trigger methods and the anti-overload methods.
\end{abstract}

Keywords: nose to tail loading state, testing method, signal processing method, storage measurement

\section{Introduction}

Projectile launched in nose to tail state is aimed to improve weapon's shooting frequency. The shooting frequency is one of the most important indicators to evaluate the weapon's power. There are two methods to increase the shooting frequency. One is using Gatling Weapon, and the other is launching projectiles with nose to tail loading state. In China, the Gatling rapid-fire gun with the multiple tube diameter of $30 \mathrm{~mm}$ has a firing rate of 10,000 rounds per minute.

The basic principle of the nose to tail loading state is putting projectiles nose to tail in one gun barrel with propellant packed between the two adjacent projectiles, and the projectiles will be fired one by one with the ignition of propellant controlled by an electronic system [1]. The shooting frequency can be increased by shortening the interval time of ignition. For example, if the interval time of the ignition is $4 \mathrm{~ms}$, the firing rate of the weapon can reach up to 15000 rounds per minute. Furthermore, the firing rate can be even higher under the multiple volley way.

In nose to tail loading state, it may occur that the projectile's motion time in bore is shorter than the interval time of ignition, which may result in a phenomenon that two or three projectiles may exist in one gun barrel simultaneously. For a certain projectile except the first one, the motion is not only 
affected by its propellant gas pressure (the base pressure), but also influenced by the gas pressure from the launch of previous projectile (the nose pressure). The peak value of the base pressure may reach up to $400 \mathrm{MPa}$, and the value of the nose pressure depends on the base pressure of the previous projectile. For example, if the motion time of the projectile in single launched state is $6 \mathrm{~ms}$ and the interval time is $4 \mathrm{~ms}$, the second projectile's peak value of the nose pressure will be up to $150 \mathrm{MPa}$. Since the mechanical characteristics and the motion characteristics of the projectile in nose to tail loading state are different from them in the single launched state in bore, weapons' final shooting accuracies will be different as a result. In conclusion, getting the interior ballistics parameter of projectile is quite important to analyze the projectiles' interior ballistics motion characteristics in nose to tail loading state. There are two methods including the numerical simulation and the experimental test to get the interior ballistics parameter. However, the experimental test method is more important because it can test the relative parameters directly.

According to the analysis of the projectile's interior ballistics characteristics in nose to tail loading state, the base pressure and the nose pressure are the main forces for the projectile to move in the interior ballistics. In addition, the motion acceleration reflects the motion characteristic of the projectile in the chamber. Therefore, it is an effective technical means to study the projectile's interior ballistics characteristics by testing the base pressure, the nose pressure and the motion acceleration of the projectile.

Microwave interferometry [2] is a common approach to test the projectile's interior ballistics velocity. With this method, microwave is radiated towards the projectile moving in bore and the Doppler signal reflected from the projectile will be received. The displacement- time relation and the velocity - time relation can be obtained by processing the relevant Doppler signal. Besides, the acceleration variation can be obtained by differentiating the velocity- time curve. Since the direction of microwave is not parallel to the bore axis, there is a measuring blind area which may lead to a failure to get the whole stroke motion of the projectile especially the motion parameter of the squeeze period. Furthermore, if two projectiles are moving in one gun barrel simultaneously, this method can only test the first one's motion parameter in chamber. Accordingly, Microwave interferometry can't be used to test the motion parameter of the projectile in nose to tail loading state.

Copper Cylinder manometry [3] is used to measure the base pressure of projectile. Although this method can measure the maximum base pressure, it can't acquire the full pressure test curve which may lead to a difficulty to know the variation of the base pressure during the whole projectile's motion period.

Storage measurement method [4] is another way to test the interior ballistics parameters of projectile. Under the premise that the projectile's ballistics motion characteristic is not changed, the projectile will be launched with the miniature data acquisition and storage device putting into the simulation test projectile, then the information can be acquired and stored by the data acquisition and storage device in real-time. When the experiment is finished, we recover the simulation test projectile, and the data will be uploaded, processed and reproduced by a computer in the end. This method is widely used in the test of the base pressure and the three-dimensional acceleration of the projectile in single launched state [5].

In our study, the projectile with a diameter of $30 \mathrm{~mm}$ has a smaller size. It is essential to test the base pressure and the nose pressure at the same time in nose to tail loading state as well. However, there are no test methods and test devices to 
test the interior ballistics parameters of projectile in nose to tail loading state until now.

This paper proposes a storage measurement method to test the interior ballistics parameters of a $30 \mathrm{~mm}$ diameter projectile in nose to tail loading state. We design the structure of the simulation test projectile, clarify the function of all parts of the simulation test projectile, design the circuit module of storage measurement device. We establish the pressure measurement model based on the inertial force correction, with the tested parameters and the established correction model, we can achieve the variation of base and nose pressure. In the end of paper, we discuss the key technologies in the design progress of the storage measurement device.

The research of the test method is a practical way to access projectile's base and nose pressure variation, and it has a certain feasible significance for obtaining the projectile interior ballistics characteristics in nose to tail loading state as well as evaluating weapon's performance index.

\section{Overall Scheme Design of the Parameters of the Projectile Motion Test}

According to the analysis of the interior ballistics characteristics of the projectile in nose to tail loading state, the test indicators are shown in Table 1.

Table 1. Test Indicators

\begin{tabular}{|l|l|}
\hline Base Pressure & $400 \mathrm{MPa}$ \\
\hline Nose Pressure & $150 \mathrm{MPa}$ \\
\hline Motion Acceleration & $60000 \mathrm{~g}$ \\
\hline Effective Measurement Time & $10 \mathrm{~ms}$ \\
\hline
\end{tabular}

Overall scheme of the storage measurement device is shown in Figure 1. The simulation test projectile is designed with the same shape and length as the actual one. The test sensors and test circuit module are installed in the simulation test projectile to finish packaging the whole device storage measurement. The filler material is put into the projectile to make sure that the device has the same mass and centroid position as the actual one. When the device is moving in bore, the parameters during the motion period can be measured and stored.

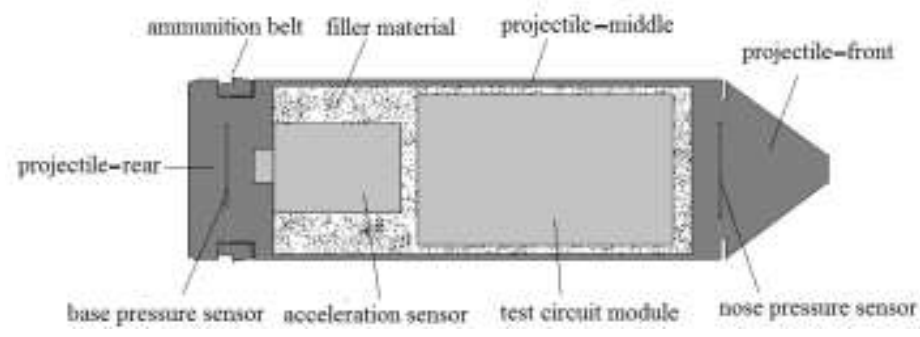

Figure 1. Overall Scheme of Storage Measurement Device

\subsection{Design of the Simulation Test Projectile}

The size of the simulation test projectile is limited by the inherent length and diameter of the $30 \mathrm{~mm}$ diameter projectile. In addition, cavity of simulation test projectile will be installed with test circuit module, leading to the decrease of test sensors' installation space. Therefore, film gauge is selected to test the force caused by the propellant gas. This kind of sensor is more suitable in the situation due to its thinner thickness and smaller volume. In addition, piezoelectric crystal transducer is chosen to test the motion acceleration of projectile.

With the overall scheme of the storage measurement device shown in Figure 1, the structure of simulation test projectile is composed of 4 parts: projectile-front, projectile- 
middle, ammunition belt and projectile-rear. Two pressure sensors are stuck on the front surface and on the back surface of the projectile-middle respectively. The projectile-front and projectile-rear are connected to the front and the back of the projectile-middle by bolts respectively as well. The force acting on the projectile-front will be passed to the nose pressure sensor and the force acting on the projectile-rear will be passed to the base pressure sensor. The acceleration sensor is connected to the inner wall of the projectile-middle by a bolt rigidly. The test circuit module is also installed in the projectile-middle. The remaining space of the projectile-middle is filled with potting material to fix the test circuit module and cushioning material to reduce the peak overload. The ammunition belt is designed to ensure a close contact between the projectile and the barrel rifling. It can also prevent the leakage of powder gases from the surrounding of the projectile.

\subsection{Design of the Test Circuit Module}

The test circuit module is installed in the projectile-middle to acquire and store the information. Schematic design of the test circuit module is shown in Figure 2.

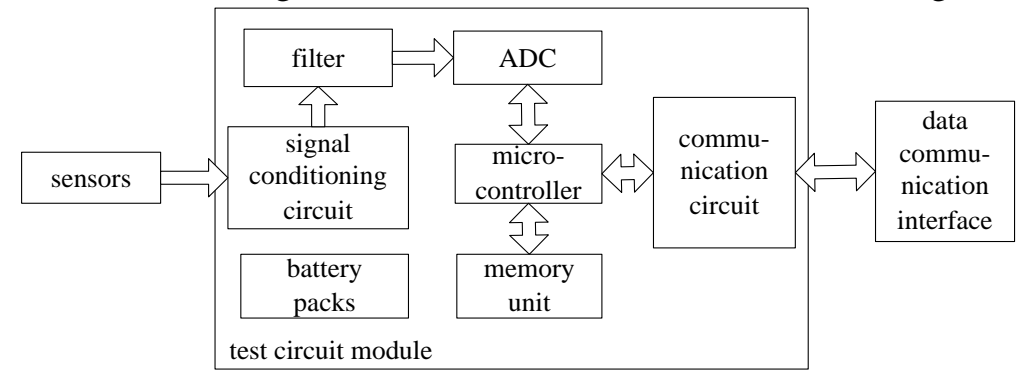

Figure 2. Schematic Design of Test Circuit Module

The module is composed of signal conditioning circuit, filter, ADC, microcontroller, memory unit, communication circuit and battery packs. The sensors' output signals are sent into the signal conditioning circuit by low noise and high strength wires. The output of the signal conditioning circuit is a voltage signal which is proportional to the relevant measured signal. Then the high frequency interference of the signal will be filtered out by a filter circuit. ADC is designed to acquire the signal and change the signal from analog to digital with the help of the microcontroller. At last, the digital signal is stored into memory unit and it will be uploaded to a PC by the communication circuit after the recovery of storage measurement device.

\section{Establishment and Analysis of the Pressure Measurement Model}

\subsection{Analysis of the Pressure Gauge Selection}

To obtain the base pressure and the nose pressure, two film force gauges are chosen to test the forces act on each of their faces according to the mechanical structure discussed in part 2. There are two types of film force gauges including the strain gauge and the piezoelectric gauge. However, the strain gauge has bad characteristics in the high-frequency band. This kind of gauge is not suitable for testing propellant gas force which has a wide effective bandwidth.

Polyvinylidene fluoride (PVDF) is a new kind of piezoelectric material with strong piezoelectric properties as well as good mechanical flexibility and small size [6-7]. It can be used to make a thin film sensor with the thickness less than $100 \mu \mathrm{m}$. As a result, the PVDF piezoelectric sensor is selected in our study. 


\subsection{Pressure Measurement Model}

Two selected PVDF piezoelectric sensors will move together with the simulation test projectile which is accelerated by the action of the base pressure and the nose pressure. The output of the sensor is influenced by the inertial forces caused by some parts of the simulation test projectile [8]. Therefore, it is necessary to research the relationship between the output of the sensor and the actual pressure.

The simulation test projectile is made of the material with high strength and high stiffness, for this reason, the projectile-front, projectile-middle and projectile-rear can be regarded as rigid body. Since the projectile only moves along with the axis of the gun, there is no need for us to take its rotational movement into consideration. The simplified mounting structure model of the pressure sensors and the simulation test projectile is shown in Figure 3.

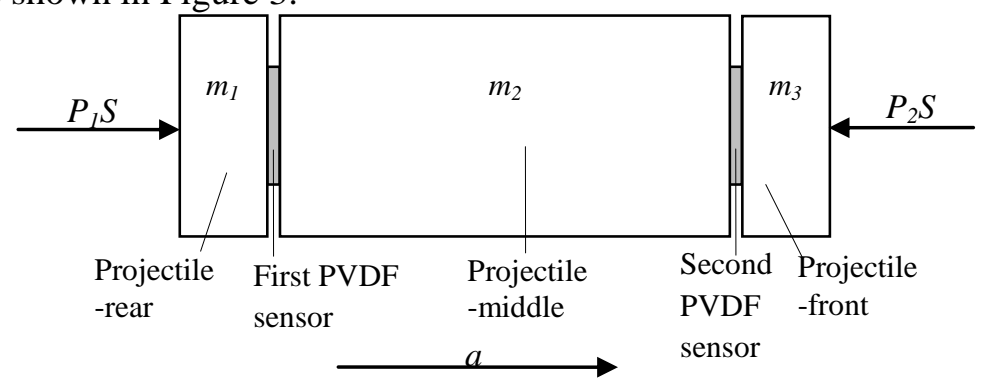

Figure 3. Simplified Mounting Structure Model

In Figure 3, $a$ is the projectile's motion acceleration and the right direction is defined as the positive direction. $P_{1}$ and $P_{2}$ are the base pressure and the nose pressure of projectile respectively. $S$ is the effective area acted by the propellant gas on the projectile. The output of the PVDF sensor is the force acting on its surface. Assuming that $F_{x}$ is the force acting on the first PVDF sensor and $F_{y}$ is the force acting on the second PVDF sensor. The mass of the projectile-front, the projectile-middle, and the projectile-rear are $m_{1}, m_{2}$ and $m_{3}$ respectively. The mass of the PVDF sensor can be ignored because it is far lighter than the other parts of the model. According to Newton's third law, the relationship among the base pressure, the nose pressure and the acceleration is:

$P_{1} S-P_{2} S=\left(m_{1}+m_{2}+m_{3}\right) a$

To study the force acting on the first PVDF sensor, we regard the projectilemiddle, the second PVDF sensor and the projectile as an equivalent mass block whose mass is $m e_{2}$, where $m e_{2}=m_{2}+m_{3}$. The result of the force analysis for equivalent mass block is shown in Figure 4.

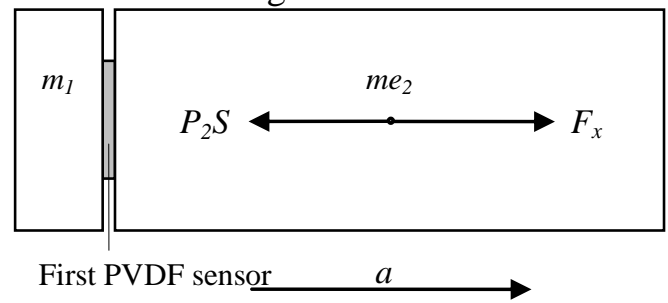

Figure 4. Force Analysis for Equivalent Mass Block

$F_{x}$, the reaction force from the first PVDF sensor and $P_{2} S$, the nose pressure from the propellant gas both are acting on the equivalent mass block 
synchronously. Therefore, the mass block moves with the acceleration $a$, and the relationship among them is:

$$
F_{x}-P_{2} S=m e_{2} \cdot a
$$

Similarly, to study the force acting on the second PVDF sensor, we regard the projectile-middle, the second PVDF sensor and the projectile as an equivalent mass block whose mass is $m e_{1}$, where $m e_{1}=m_{1}+m_{2}$. The result of the force analysis for projectile-front is shown in Figure 5.

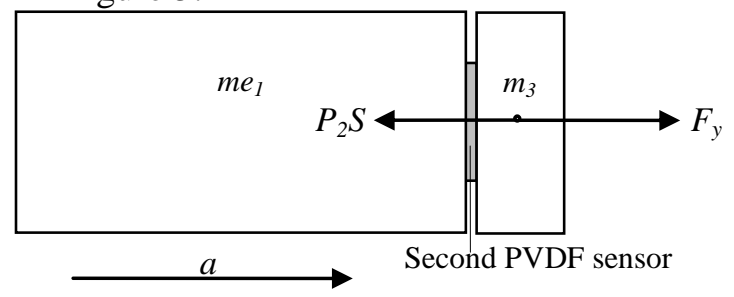

Figure 5. Force Analysis for Projectile-Front

$F_{y}$, the reaction force from the second PVDF sensor and $P_{2} S$, the nose pressure from the propellant gas are acting on the projectile-front synchronously. Therefore, the projectile-front moves with the acceleration $a$, and the relationship among them is:

$$
F_{y}-P_{2} S=m_{3} \cdot a
$$

We can calculate $P_{2}$ by considering formula (1) and (2):

$P_{2}=\frac{1}{2 S}\left[F_{x}+F_{y}-\left(m_{2}+2 m_{3}\right) a\right]$

Substitute (4) into (1), $P_{l}$ can be calculated as:

$P_{1}=\frac{1}{2 S}\left[F_{x}+F_{y}+\left(m_{2}+2 m_{1}\right) a\right]$

It is obvious that the PVDF sensors' output is related to the mass blocks' inertial force acting on the sensors. To obtain the value of the inertial force, the acceleration should be measured by the acceleration sensor. And then we can calculate the base pressure $P_{1}$ and the nose pressure $P_{2}$ by formula (4) and (5).

\section{Design of the Test Circuit Module of the Storage Measurement Device}

\subsection{Signal Conditioning Circuit}

The outputs of the PVDF sensor and the acceleration sensor are all electric charge signals. The piezoelectric sensor has extremely high insulation resistance to make sure that the electric charge will not leak. The charge amplifier, a circuit with high input resistance and low output resistance, is the dedicated preamplifier circuit to process electric charge signals from the piezoelectric sensor. The output voltage of the charge amplifiers is proportional to the input electric charge signal. The charge amplifier circuit is shown in Figure 6. 


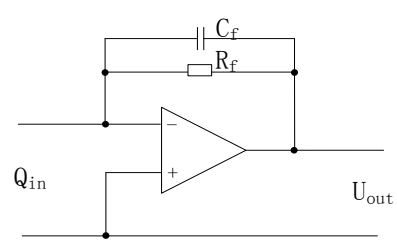

Figure 6. Charge Amplifier Circuit

The capacitor $C_{f}$ and the resistor $R_{f}$ are placed between the inverting input terminal and the output terminal of the op amp in parallel. The relationship between the input charge signal and the output voltage signal is:

$U_{\text {out }}=-Q_{\text {in }} / C_{f}$

Where $R_{f}$, a high value resistor, is used to provide direct feedback and reduce the zero drift of the amplifier's output, making the charge amplifier stable.

The interference signal outside the effective bandwidth will be filtered by the filter circuit. Since the effective bandwidth of the measured signal in chamber is $5 \mathrm{kHz}$, the upper cut-off frequency of the filter circuit is set slightly larger than $5 \mathrm{kHz}$.

\subsection{ADC Circuit}

The ADC circuit is designed to change the analog voltage signal generated by the signal conditioning circuit into a digital signal which has a stronger antiinterference ability. According to the Sampling Theorem and the actual engineering situation, we can assume that:

$f_{s} \geq(10 \sim 20) f_{e b}=(50 \sim 100) \mathrm{kHz}$

Where $f_{s}$ is the ADC's sampling frequency and $f_{e b}$ is the measured signal's effective bandwidth. That is to say, $100 \mathrm{kHz}$ is a reasonable sampling frequency for ADC.

The core of the analog to digital conversion circuit is the ADC chip. The AD7324 is a 4-channel, 12-bit plus sign, successive approximation ADC. It can accept true bipolar analog input signals. The ADC has a high speed serial interface that can operate at a throughput rate up to 1 MSPS which can meet the requirement of testing the base pressure, the nose pressure and the acceleration synchronously. Furthermore, the chip has a SPI serial interface and only needs 4 signal lines to communicate with other device, thus simplifying the design of the PCB layout greatly.

\subsection{Microcontroller and Signal Storage Circuit}

The 32 bit STM32F1 Series MCU based on ARM-Cortex-M3 core is used as the microcontroller. The chip has a SPI serial interface which is quite convenient for the communication between the microcontroller and the ADC.

The signal storage circuit is used to store the digital signal converted by the ADC. If the sampling frequency is set as $200 \mathrm{kHz}$ per channel, the sampling time is set as $15 \mathrm{~ms}$ and the 12-bit plus sign output of ADC costs two bytes each time, then the storage space required for the whole test is:

$200 \mathrm{kHz} \cdot 15 \mathrm{~ms} \cdot 2$ Byte $\cdot 3=18 \mathrm{kByte}$

Since a 512KB Flash memory is integrated on the microcontroller, the memory unit can be replaced by the on chip Flash. This method can simplify the design of the circuit. 


\section{Research on Key Technologies of the Storage Measurement Device}

Since the storage measurement device acquires and stores signals in the chamber of the gun, the whole device should be able to withstand harsh environment, including high overload, high shock and vibration, instantaneous high temperature and pressure. Therefore, researching on the key technologies of the device is very important to get the success of the measure experiment.

\subsection{Mechanical Design of the Pressure Test}

The projectile-front and the projectile-rear of the simulation test projectile are the core parts during the period of pressure measurement. Their mechanical parts contact with the propellant gas pressure directly and then convert the pressure into force which act on each surface of pressure sensors. These pressure-forceconverting parts should have suitable strength and stiffness as a result.

In our design, the pressure-force-converting parts are made of $35 \mathrm{CrMnSi}$, a kind of steel with high strength and low alloy. Yield strength of the material is $1275 \mathrm{MPa}$ and elastic modulus is $195 \mathrm{GPa}$. The structure of the projectile-rear is shown in Figure 7(a). When the propellant gas pressure acts on the surface of the pressure-force-converting part, only a slight elastic deformation will occur and it won't cause structural damage to the pressure-force-converting part.

Film gauge is used to test the pressure passed by the pressure-force-converting parts. To guarantee the accuracy of the result, the force converted by the pressure-forceconverting part should act on the surface of sensor completely. The schematic design of the pressure test is shown in Figure 7. The film gauge is stuck on the surface of the projectile-middle. The pressure-force-converting part and the projectile-middle are connected by three bolts closely to apply a certain preload to the film gauge. When the propellant gas pressure acts on the pressure-force-converting part, the pressure can be passed to the sensor directly and successfully due to the close contact between the pressure-force-converting part and the sensor.

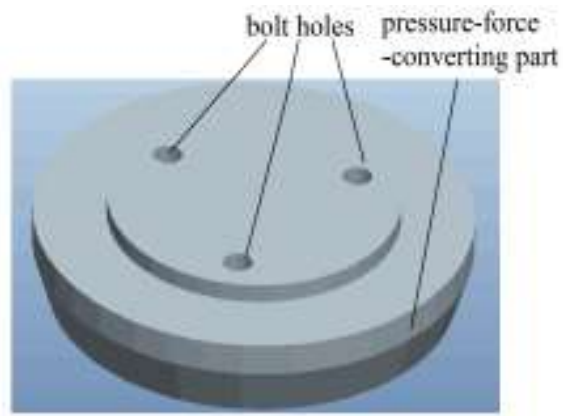

(a) Structure of Projectile-rear

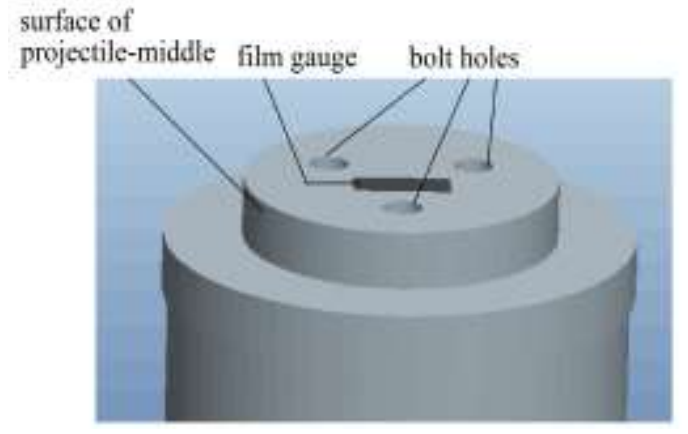

(b) Structure of Projectile-middle

Figure 7. Schematic Design of Pressure Test

\subsection{Miniaturization of the Test Circuit Module}

In order to make sure certain strength and stiffness of projectile, the projectile should have some thickness of the wall. With the small volume of the simulation test projectile, the space reserved for the test circuit module is much limited. As a result, miniaturization of the test circuit is an important problem to be solved. The specific measures to solve the problem are listed as followed:

1. Using micro-packaged electronic components. For example, using resistors and capacitors with 0402 SMD package, using chips with TSSOP package which has intensive pins or use chips with BGA, WSON packages whose pins are located below the chip. 
2. Using the design idea that stacking multiple circuit boards together. Since it is hard to layout all electronic components in one PCB board, we can reserve the interface with same position in several PCB boards and layout all components firstly, and then splice the boards with plugs and receptacles.

\subsection{Trigger Method}

The signals need to be measured only last for several milliseconds. Besides, the testing system works on battery power and the test circuit module should be powered on before being put in the chamber of the gun. However, the time interval of the projectile from being loaded to being launched is not definite. As a result, there will be a waste of power if the test circuit module starts to work as soon as being powered on. The trigger technique is used to solve the problem and extend the batteries' life greatly. There have been two kinds of trigger methods as followed in our study:

1. Software Trigger. If the test circuit module works in this way, one channel's data acquisition should always be on working before the launch of the simulation test projectile. The channel can be set to test the pressure or the acceleration. As soon as the data acquired is larger than the threshold which is set before the test, it shows that the simulation test projectile is being launched. Then the test circuit module should open all channels to start data acquisition immediately.

2. Hardware trigger. A wire is used in this method to connect the ignition control terminal and the trigger input port of the test circuit module. The signal of ignition can be regarded as a trigger signal. When the microcontroller detects the trigger signal, the test circuit module will start to acquire and store the measured signals immediately.

Threshold used in software trigger method doesn't have an explicit physical meaning to correspond with the projectile's ballistics stage. The sampling zero point in hardware trigger is the moment of ignition, and the measured signals have explicit relationship with the time. However, a trigger wire is needed in this method, and the trigger process will be failed if the trigger wire snapped before the formal test.

\subsection{Anti-Overload Technologies}

Deformation of board, shedding of solder joints and failure of electronic components are often happened when the test circuit module are in high overload. These phenomena may reduce the success rate of the test seriously. Anti-overload technologies are needed to protect the test circuit module and reduce the real bearer of the board [9].

An effective anti-overload method is to combine the material potting and off-load components installation. Firstly, we put the potting material and the test circuit module together to make them stuck and solidified as an entirety to prevent the PCB boards from damage in shock and vibration. Secondly, we cover the entirety with load shedding material such as spring and aluminum foam [10]. Therefore, the peak value of the overload on the test boards could be reduced.

\section{Conclusion}

According to the mechanical characteristics and motion characteristics of the projectile in nose to tail loading state, this paper proposes a storage measurement method to test the projectile's interior ballistics parameters, including the base pressure, the nose pressure and the motion acceleration. The pressure measurement model with inertial force correction is established under the installation between the pressure sensors and the simulation test projectile. The model shows that the output of the sensor is related with the base pressure, the nose pressure, as well as the inertial force caused by the mass block's mass and 
the motion acceleration. With the pressure measurement model, we can calculate the base pressure and the nose pressure. The test circuit module is designed to acquire and store the measured signals. In the end of this paper, several key technologies in the design progress of the storage measurement device are discussed. Consequently, the contents studied in this paper will offer a practical testing method to measure the interior ballistics parameters of the projectile in nose to tail loading state.

\section{References}

[1] H. L. Yu, X. T. Rui, F. F. Yang, G. Wang and Z. J. Liu, "Modeling and Simulation of Launch Dynamics for "Metal Storm" Weapon", Journal of Nanjing University of Aeronautics \&Astronautic, vol. 42, no. 5, (2010), pp. 574-577.

[2] Y. C. Zhang, J. B. Zhang and W. R. Yan, "Burning Rules of Gun Propellant in Powder Chamber Based on Bullet Velocity Measurement with Microwave Interferometer", Chinese Journal of Explosives \& Propellants, vol. 33, no. 4, (2010), pp. 74-77.

[3] Y. Zhang, J. Zu, H. Y. Zhang and D. W. Shen, "Research on Projectile Breech Pressure Measuring Method Based on Storage Measurement Technique", Fire Control \& Command Control, vol. 39, no. 1, (2014), pp. 172-179.

[4] M. Li and M. J. Liu, "Research on Missile-borne storage measurement system", Measuring Technology and Mechatronics Automation, ICMTMA'09. International Conference, vol. 1, (2009), pp. 326-329.

[5] H. J. Liu, D. W. Shen, X. E. Li and L. Zhuo, "Technical Analysis of Missile-borne High-g Three-axis Acceleration Test System", CHINESE JOURNAL OF SENSORS AND ACTUATORS, vol. 26, no. 1, (2013), pp. 21-24.

[6] X. J. Ren and H. L. Chen, "Application of PVDF sensors in shock wave measurement of near zone of explosion", JOURNAL OF VIBRATION AND SHOCK, vol. 31, no. 12, (2012), pp. 146-149.

[7] A. V. Shirinov and W. K. Schomburg, "Pressure sensor from a PVDF film", Sensors and Actuators A: Physical, vol. 142, no. 1, (2008), pp. 48-55.

[8] D. W. Pohl, "Dynamic piezoelectric translation devices", Review of Scientific Instruments, vol. 58 no. 1, (1987), pp. 54-57

[9] F. Wen and J. Z. Qiao, Y. Li, "Study on anti over-loaded technology in high over-loaded storage testing", Transducer and Microsystem Technologies, vol. 28, no. 9, (2009), pp. 31-33.

[10] O. B. Olurin, K. Y. G. McCullough and N. A. Fleck, "Fatigue crack propagation in aluminium alloy foams", International Journal of Fatigue, vol. 23, no. 5, (2001), pp. 375-382.

\section{Authors}

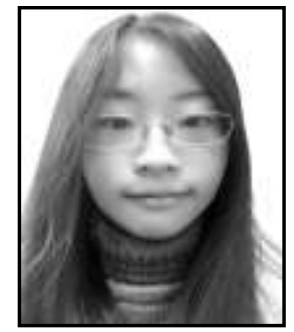

Hang Yu, she is a Ph.D. student in the School of Mechanical Engineering, Nanjing University of Science \& Technology. Her research interests include dynamic parameter test of weapons and signal processing.

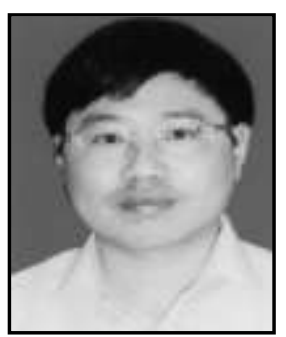

Kong Deren, he is a doctoral supervisor in the School of Mechanical Engineering, Nanjing University of Science \& Technology, Nanjing, China. His research interests include modern measuring theory and application, modern sensor technology and systems. 